University of Wollongong

Research Online

Faculty of Informatics - Papers (Archive)

Faculty of Engineering and Information

Sciences

18-10-2006

\title{
Adaptive overlap-add equalization for MB-OFDM ultra-wideband
}

\author{
Darryn Lowe \\ University of Wollongong, darrynl@uow.edu.au \\ Xiaojing Huang \\ University of Wollongong, huang@uow.edu.au
}

Follow this and additional works at: https://ro.uow.edu.au/infopapers

Part of the Physical Sciences and Mathematics Commons

\section{Recommended Citation \\ Lowe, Darryn and Huang, Xiaojing: Adaptive overlap-add equalization for MB-OFDM ultra-wideband 2006. https://ro.uow.edu.au/infopapers/405}

Research Online is the open access institutional repository for the University of Wollongong. For further information contact the UOW Library: research-pubs@uow.edu.au 


\title{
Adaptive overlap-add equalization for MB-OFDM ultra-wideband
}

\author{
Abstract \\ A zero-pad can be used with orthogonal frequency division multiplexing (OFDM) for low-complexity \\ robustness against multipath interference. In this paper, we use adaptive overlap-add (OLA) equalization \\ for improvements of up to $0.6 \mathrm{~dB}$ improvement when used with multi-band OFDM (MB-OFDM) ultra- \\ wideband in multipath channels. A theoretical model that relates the size of OLA window to post- \\ equalizer signal-to-noise ratio is derived. An approximating algorithm is then developed that is suitable for \\ low-complexity implementation, with Monte Carlo simulations used to quantify the performance \\ improvements. We conclude that adaptive OLA equalization is computationally simple and can be \\ implemented while remaining fully compliant with the MB-OFDM standard.

\section{Keywords} \\ UWB, ultra-wideband, MB-OFDM, equalization, overlap-add, OLA \\ Disciplines \\ Physical Sciences and Mathematics

\section{Publication Details} \\ This paper was originally published as: Lowe, D \& Huang, X, Adaptive overlap-add equalization for MB- \\ OFDM ultra-wideband, 2006 International Symposium on Communications and Information Technologies \\ (ISCIT), Bangkok, Thailand, 18-20 October 2006. Conference information is available here.
}




\title{
Adaptive Overlap-Add Equalization for MB-OFDM Ultra-Wideband
}

\author{
Darryn Lowe and Xiaojing Huang \\ School of Electrical, Computer and Telecommunications Engineering \\ University of Wollongong \\ Wollongong, Australia, 2522 \\ Email: \{darrynl, huang\}@uow.edu.au
}

\begin{abstract}
A zero-pad can be used with orthogonal frequency division multiplexing (OFDM) for low-complexity robustness against multipath interference. In this paper, we use adaptive overlap-add (OLA) equalization for improvements of up to $1 \mathrm{~dB}$ when used with multi-band OFDM (MB-OFDM) ultra-wideband. A theoretical model that relates the size of OLA window to post-equalizer signal-to-noise ratio is derived. An approximating algorithm is then developed that is suitable for low-complexity implementation, with Monte Carlo simulations used to quantify the performance improvements. We conclude that adaptive OLA equalization is computationally simple and can be implemented while remaining fully compliant with the MB-OFDM standard.
\end{abstract}

\section{INTRODUCTION}

Ultra-wideband (UWB) has tremendous potential for highrate low-power communication due to its high data rates and resistance to interference [1]. With UWB officially defined in 2002 by the United States Federal Communications Commission (FCC) as a signal with a $10 \mathrm{~dB}$ bandwidth of at least $500 \mathrm{MHz}$ and a maximum equivalent isotropic radiated power spectral density (PSD) of no more than $-41.3 \mathrm{dBm} / \mathrm{MHz}$ in the $3.1-10.6 \mathrm{GHz}$ band [2], the race is on to exploit this untapped spectral resource.

The first UWB technology to obtain international standardization is multi-band orthogonal frequency division multiplexing (MB-OFDM) [3][4] developed by the WiMedia Alliance. The MB-OFDM standard defines both an UWB physical layer (PHY) and medium access control (MAC) and supports data rates from $53.3 \mathrm{Mb} / \mathrm{s}$ to $480 \mathrm{Mb} / \mathrm{s}$. MB-OFDM divides the several gigahertz of spectrum allocated by the FCC into 14 bands, each with a $528 \mathrm{MHz}$ bandwidth. These bands are then bundled into 5 band groups with only the first defined as mandatory.

MB-OFDM, which shares the generic OFDM block diagram of Fig. 1, has four distinguishing characteristics with regard to previous OFDM wireless local area network (WLAN) standards, such as IEEE 802.11 a/g and HiperLAN/2. First, an MB-OFDM symbol is comprised of 128 samples rather than the 64 samples used in IEEE 802.11a. Second, a zero-pad (ZP) is used rather than a cyclic prefix $(\mathrm{CP})$. Although a $\mathrm{ZP}$ results in higher peak-to-average power, it is more efficient than a $\mathrm{CP}$ since the energy of the $\mathrm{CP}$ is discarded by the receiver. Third, MB-OFDM supports a range of optional diversity improvements. This includes frequency domain spreading

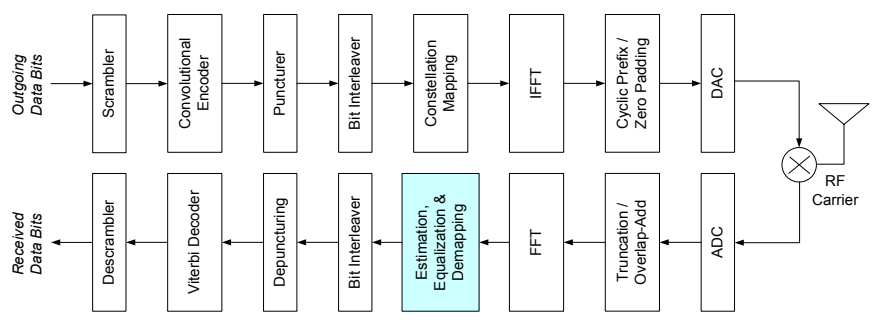

Fig. 1. Block diagram of generic OFDM transmitter.

(FDS) and time domain spreading (TDS), both of which offer an extra $3 \mathrm{~dB}$ of process gain when activated, as well as dual carrier modulation (DCM) to combat frequency selective fading at high data rates. Fourth, time-frequency codes (TFCs) support optional time-frequency interleaving (TFI) to permit up to a $4.7 \mathrm{~dB}$ increase in transmit power. This is possible since when each $528 \mathrm{MHz}$ band is only active for $1 / 3$ of the time, the power radiated when it is on can be up to 3 times higher without violating the $-41.3 \mathrm{dBm} / \mathrm{MHz}$ FCC limit.

The spread of data rates offered by MB-OFDM allows efficient communication over distances from less than $10 \mathrm{~cm}$ to well over $10 \mathrm{~m}$. In short low-loss channels, which may even see sender and receiver physically touching, there will often be clear line-of-sight (LOS), nominal delay spread and little path loss. This almost Gaussian channel will therefore support very high data rates. However, when sender and receiver are further apart and/or do not have LOS, the received signal may contain significant multipath components. In such an adverse channel, only low data rates will be possible.

The focus of this paper is on how the receiver baseband signal processing can adapt the size of the overlapadd (OLA) window used during channel equalization based on measured channel delay spread. To that end, Section II considers the theoretical relationship to derive an expression that is broadly applicable to any ZP OFDM system. As MB-OFDM transceivers must be efficient at both short and long range, Section III then develops an MB-OFDM-specific OLA equalizer that is computationally inexpensive and fully compliant with the standard. The performance is then analyzed via Monte Carlo simulations in Section IV and the findings summarized in the conclusions of Section V. 


\section{THEORY}

\section{A. Zero-Pad OFDM}

OFDM systems modulate coded information bits, pilots and guards to a block of $N$ parallel sub-carriers. If we denote ${ }^{1}$ the $N \times 1$ vector of symbol constellations modulated to the $i^{\text {th }}$ OFDM block as $\mathbf{C}(i)$, then the corresponding time-domain vector is

$$
\mathbf{x}(i)=\mathbf{W}^{H} \mathbf{C}(i)
$$

where $\mathbf{W}$ is the $N \times N$ FFT matrix with $[\mathbf{W}]_{k, n}=$ $\frac{1}{\sqrt{N}} e^{-j 2 \pi k n / N}$. In ZP OFDM, the time-domain OFDM symbol is postfixed with $L$ samples of $\mathrm{ZP}$ to bring the total samples per transmitted block to $P=N+L$.

Consider a multipath channel with a complex baseband equivalent channel impulse response (CIR) of $h(n)$ that we denote as a $P \times 1$ vector $\mathbf{h}=[h(0), h(1), h(2), \ldots, h(P-1)]^{T}$. Assuming that the CIR is less than $L$ samples in duration, i.e. $\sum_{n=L+1}^{\infty}|h(n)|^{2}=0$, the ZP will completely remove all interblock interference (IBI). As each block is independent, we omit the block indices from this point onward for convenience.

As the over-the-air transmission will be convolved with the CIR, the signal at the input to the receiver digital baseband is

$$
\mathbf{s}=\mathbf{H} \mathbf{x}+\mathbf{z}
$$

where $\mathbf{H}$ is a $P \times N$ tall Toeplitz matrix with $\mathbf{h}$ as its first column, i.e.

$$
\mathbf{H}=\left[\begin{array}{cccc}
h(0) & 0 & \cdots & 0 \\
h(1) & h(0) & \cdots & 0 \\
\vdots & \vdots & \ddots & \vdots \\
h(N-1) & h(N-2) & \cdots & h(0) \\
\vdots & \vdots & \vdots & \vdots \\
h(P-1) & h(P-2) & \cdots & h(P-L)
\end{array}\right]
$$

and $\mathbf{z}$ represents $\mathrm{AWGN}$ as a $P \times 1$ vector of Gaussian distributed random variables of variance $\eta^{2}$. To estimate the transmitted signal, we can denote an ideal equalizer as

$$
\hat{\mathbf{x}}=\mathbf{H}^{\dagger} \mathbf{s}=\mathbf{x}+\mathbf{H}^{\dagger} \mathbf{z}
$$

Equation (4) can be viewed as an over-constrained least squares problem [5]. As the sensitivity to $\mathbf{z}$ is proportional to the condition number of the matrix $\mathbf{H}$ [6], the estimation error of an ideal equalizer is bounded by

$$
\frac{\left\|\mathbf{H}^{\dagger} \mathbf{s}-\mathbf{x}\right\|}{\|\mathbf{x}\|} \leq \frac{2 \kappa(\mathbf{H})}{\sqrt{\gamma_{i n}}}
$$

where $\gamma_{i n}$ is the signal-to-noise ratio (SNR) at the equalizer input and $\kappa(\mathbf{H})$ is the condition number of matrix $\mathbf{H}$, i.e. the ratio between the maximum and minimum values of the singular value decomposition (SVD).

\footnotetext{
${ }^{1}$ Throughout this paper, the following matrix notation conventions are adopted: |.| and ||.|| denote the 1-norm and 2-norm respectively; [.] ${ }^{T}$ and $[.]^{H}$ the transpose and Hermitian transpose respectively; $[.]_{k, n}$ the element of the $k^{\text {th }}$ row and $n^{\text {th }}$ column; [.] $]^{-1}$ and [.] $]^{\dagger}$ the matrix inverse and pseudoinverse respectively.
}

The SNR at the input to the equalizer can be derived from (2) as

$$
\gamma_{i n}=\frac{\|\mathbf{H} \mathbf{x}\|^{2}}{\|\mathbf{z}\|^{2}}
$$

and the SNR after equalization can be derived from (4) as

$$
\gamma_{\text {out }}=\frac{\|\mathbf{x}\|^{2}}{\left\|\mathbf{H}^{\dagger} \mathbf{z}\right\|^{2}}=\frac{\|\mathbf{x}\|^{2}}{\left\|\mathbf{H}^{\dagger} \mathbf{s}-\mathbf{x}\right\|^{2}}
$$

By using the bound defined in (5), we can conclude that

$$
\gamma_{\text {out }}=\frac{\|\mathbf{x}\|^{2}}{\left\|\mathbf{H}^{\dagger} \mathbf{s}-\mathbf{x}\right\|^{2}} \geq \frac{\gamma_{\text {in }}}{4 \kappa^{2}(\mathbf{H})}
$$

Recall now that an OFDM symbol is the sum of $N$ sinusoidal sub-carriers nominally modulated by random data. If we model the normalized data constellations as $N$ complex random variables with magnitude $\sigma^{2}$ and uniformly distributed phases between 0 and $2 \pi$, the time-domain OFDM symbol will be a vector of $N$ complex Gaussians with variance $\sigma^{2}$. We can therefore denote the received signal energy as

$$
\|\mathbf{H} \mathbf{x}\|^{2}=\sigma^{2}|\mathbf{M} \mathbf{1}|
$$

where 1 is an $N \times 1$ vector of 1's and M denotes the energy of the channel convolution matrix, i.e. $[\mathbf{M}]_{i, j}=\left|[\mathbf{H}]_{i, j}\right|^{2}$. Also, since $\mathbf{z}$ is constant over the entire symbol period, we can state that

$$
\|\mathbf{z}\|^{2}=P \eta^{2}=(N+L) \eta^{2}
$$

By now substituting $(6,9,10)$ into $(8)$, we can define a bound on the SNR of the equalized signal as a function of the $\mathrm{ZP}$ length as

$$
\gamma_{\text {out }} \geq \frac{\sigma^{2}|\mathbf{M} \mathbf{1}|}{4(N+L) \eta^{2} \kappa^{2}(\mathbf{H})}
$$

\section{B. Overlap-Add Equalization}

In high-rate systems such as MB-OFDM, practical implementation mandates for computationally efficient algorithms. For example, direct realization of zero-forcing (ZF) or minimum mean squared error (MMSE) equalizers are not viable since both require matrix inversion [7]. Therefore, in many real-world systems, the only viable OFDM equalizers are ones that use the FFT to exploit that cyclic convolution in the timedomain is equivalent to component-wise multiplication in the frequency-domain.

To describe these equalizers, we begin by considering the case where a CP is used instead of a ZP. This restricts $\mathbf{H}$ to an $N \times N$ square circulant matrix that we denote as $\tilde{\mathbf{H}}$. The corresponding equalized symbol can be denoted as

$$
\hat{\mathbf{C}}=\left[\mathbf{W} \tilde{\mathbf{H}} \mathbf{W}^{H}\right]^{-1} \mathbf{W} \mathbf{s}=\mathcal{D}^{-1}(\mathbf{W h}) \mathbf{W} \mathbf{s}
$$

where $\mathcal{D}(\mathbf{W h})$ is a diagonal $N \times N$ matrix denoting the frequency response of the CIR. This means that matrix inversion is not necessary since

$$
\left[\mathcal{D}^{-1}(\mathbf{W h})\right]_{n, n}=\frac{1}{[\mathbf{W h}]_{n, 1}}
$$




\begin{tabular}{|c|l|l|}
\hline Parameter & Description & Value \\
\hline$f_{s}$ & Sampling frequency (MHz) & 528 \\
$N_{F F T}$ & Number of subcarriers & 128 \\
$N_{D}$ & Number of data subcarriers & 100 \\
$N_{P}$ & Number of pilot subcarriers & 12 \\
$N_{G}$ & Number of guard subcarriers & 10 \\
$N_{Z P S}$ & Samples in ZP & 37 \\
$N_{T F I}$ & Samples reserved for LO settling & 5 \\
$N_{S Y M}$ & Total number of samples per symbol & 165 \\
\hline
\end{tabular}

TABLE I

MB-OFDM PARAMETERS PER SYMBOL

\begin{tabular}{|c|l|l|l|l|l|l|}
\hline TFC & \multicolumn{7}{|c|}{ Band } \\
\hline 1 & 1 & 2 & 3 & 1 & 2 & 3 \\
2 & 1 & 3 & 2 & 1 & 3 & 2 \\
3 & 1 & 1 & 2 & 2 & 3 & 3 \\
4 & 1 & 1 & 3 & 3 & 2 & 2 \\
5 & 1 & 1 & 1 & 1 & 1 & 1 \\
6 & 2 & 2 & 2 & 2 & 2 & 2 \\
7 & 3 & 3 & 3 & 3 & 3 & 3 \\
\hline
\end{tabular}

TFC PATTERNS FOR BAND GROUP 1

The end result is that the receiver needs only one FFT for each OFDM symbol.

Although (12) is low complexity, it only works for a square circulant channel matrix $\tilde{\mathbf{H}}$. Since a $\mathrm{ZP}$ produces as a tall Toeplitz matrix $\mathbf{H}$, we truncate it to a square $N \times N$ matrix after using an OLA adds the bottom $l$ rows to the top $l$ rows[8]. We denote the result of this as $\hat{\mathbf{H}}_{l}$. If the OLA uses the entire $\mathrm{ZP}$, then $\hat{\mathbf{H}}_{l}=\hat{\mathbf{H}}_{L}=\tilde{\mathbf{H}}$. Conversely, if a smaller OLA truncates too much of the CIR, then the lost energy will make $\hat{\mathbf{H}}_{l}$ more singular and increase the condition number.

We can rewrite (11) as a function of $l$ to obtain

$$
\gamma_{\text {out }}(l)=\frac{\sigma^{2}\left|\mathbf{M}_{l} \mathbf{1}\right|}{4(N+l) \eta^{2} \kappa^{2}\left(\mathbf{H}_{l}\right)} \propto \frac{\left|\mathbf{M}_{l} \mathbf{1}\right|}{(N+l) \kappa^{2}\left(\mathbf{H}_{l}\right)}
$$

where $\left[\mathbf{M}_{l}\right]_{i, j}=\left|\left[\mathbf{H}_{l}\right]_{i, j}\right|^{2}$. To represent the analytically optimal OLA, we denote the $l$ yielding the highest SNR as $L_{A N A L}$.

\section{MB-OFDM}

The key parameters of the MB-OFDM standard [4] are summarized in Table I. Note that although the ZP added to each OFDM symbol by the transmitter is 37 samples, recall that the receiver is free to use or discard however many samples as desired.

The only limitation on the maximum number of ZP samples that can be used by the receiver is imposed by the choice of TFC, the definitions of which are shown in Table II. Some TFCs, such as TFC 1 and 2, change bands after every OFDM symbol. Since $N_{T F I}$ samples are reserved to allow the local oscillator to settle after a frequency hop, only 32 samples remain for processing. For other TFCs, such as TFCs 5, 6 and 7 , the frequency hopping is disabled so all 37 samples can be used. The remaining TFCs 3 and 4 change bands every two symbols; the useable portion of the $\mathrm{ZP}$ alternates between 32 and 37 samples.

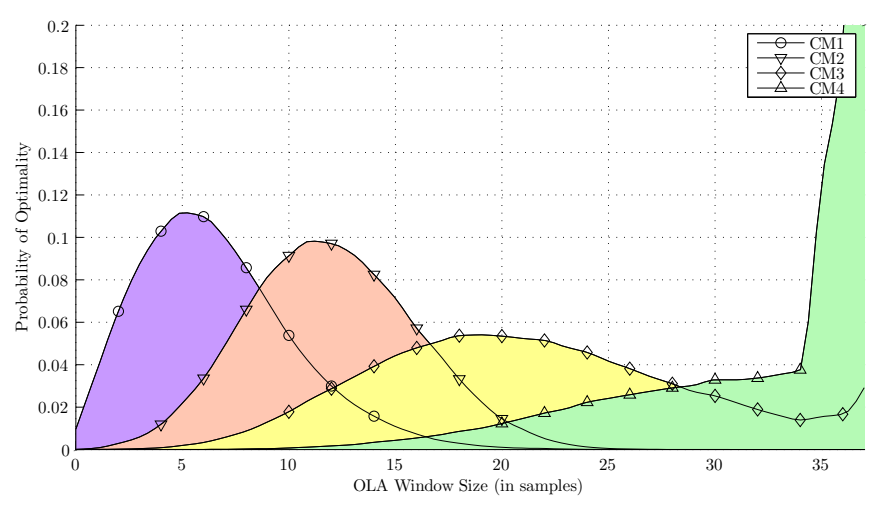

Fig. 2. Probability distribution of analytically optimal OLA sizes.

In recognition of the variety of channels that UWB devices will operate in, the IEEE 802.15.3a working group defined 4 UWB channel models, denoted as CM1 through CM4, to represent environments with delay spreads from 5 ns to 25 ns [9]. These channel models use a parameterized SalemVenezuela (S-V) model [10].

\section{A. OLA Size and Channel Model}

We now consider the distribution of the optimal OLA size for different channel models. We begin by generating 10,000 realizations of CM1 through CM4. For each realization, the optimal OLA size, from $0 \leq L \leq N_{Z P S}$, is obtained via (11). The result is shown in the probability distribution of Fig. 2. We observe that the optimal OLA size has an expected value of 5 samples in the case of CM1 and 37 samples in the case of CM4. It is also apparent that the variance in optimal ZP size increases as the CIR becomes longer.

Ideally, the receiver will estimate the optimal OLA size based on instantaneous channel conditions. Unfortunately, it is not practical to do so using (11) as this expression requires perfect knowledge of the CIR and incurs computationally expensive matrix operations such as SVD. We solve this problem in the next section by developing a simple estimation technique that reuses many of calculations that were already performed during synchronization of the packet preamble.

\section{B. Practical Estimation of Optimal ZP}

Synchronization in MB-OFDM is performed with a 128 sample training sequence $t(n)$ that is known at both transmitter and receiver. The MB-OFDM standard defines the training sequence for TFC $i$ as $t_{i}(n)$, with the preamble repeated either 24 or 12 times. The short preamble is used only when several packets are sent as a continuous stream; the long preamble is used by default.

As the receiver is not immediately aware when a transmission begins, the received training sequence can be denoted as

$$
r\left(n-n_{0}\right)=\sum_{k=0}^{L-1} t(n-k) h(k)+z(n)
$$

where $z(n)$ is AWGN with variance $\eta^{2}, h(n)$ is the CIR and $n_{0}$ is an unknown timing offset. It is the role of the receiver 
synchronization process to resolve $n_{0}$ before the end of the packet preamble. If we assume that the training sequence has an ideal auto-correlation

$$
\phi_{t t}(m)=\sum_{n=0}^{127} t(n+m) t(n)=\delta(m)
$$

then the cross-correlation between the transmitted and received signals will be

$$
\phi_{r t}\left(m-n_{0}\right)=\sum_{n=0}^{127} r\left(n+m-n_{0}\right) t(n)=\hat{h}\left(m-n_{0}\right)
$$

where $\hat{h}(n)$ is the estimated CIR with the only error due to AWGN. As the real MB-OFDM training sequences $t_{i}(n)$ do not have ideal auto-correlations, the error in $\hat{h}(n)$ will be greater than the ideal case. Fortunately, the difference will not be significant given that numerical analysis of $t_{i}(n)$ reveals that the peak energy of $\phi_{t_{i} t_{i}}(t)$ at $t=0$ is more than $20 \mathrm{~dB}$ higher than the most significant sidelobe.

To capture maximum energy in dispersive channels, the synchronization should combine the training sequence correlations over all $L$ samples of the ZP. This defines a metric

$$
p(m)=\sum_{i=0}^{L-1}\left|\phi_{r t}\left(m-n_{0}+i\right)\right|
$$

the peak of which resolves the timing offset $n_{0}$.

To estimate the optimal OLA size $L_{P R A C}$, we need to balance the extra energy captured by a larger OLA window against the increase in AWGN. We can express this relationship as

$$
\hat{\gamma}_{\text {out }}(l)=\frac{\sum_{m=0}^{l}|h(m)|^{2}}{\sum_{m=0}^{N+l}|z(m)|^{2}} \approx \frac{\sum_{m=0}^{l}\left|\phi_{t_{i} t_{i}}(m)\right|^{2}-l \eta^{2}}{(N+l) \eta^{2}}
$$

and define $L_{P R A C}$ as the $l$ for which (19) is maximized. Unlike (14), $\hat{\gamma}_{\text {out }}(L)$ can be calculated with only a few real arithmetic operations. Further, as the correlation and the summation are already preformed as part of synchronization, the incremental cost of estimating the optimal OLA size is dominated by the division operation. There are several ways by which this complexity can be reduced, including disregarding the $\eta^{2}$ in the denominator or pre-computing $1 /(N+l)$ and storing the result in a lookup table. If even greater simplifications are required, we note that the entire denominator can be approximated as $N$. This would allow the division to be replaced with a trivial binary shift.

\section{RESUlts}

We perform two types of Monte-Carlo simulations. The first considers the accuracy of the optimal OLA window estimation and the second quantifies the performance improvements in terms of PER. The simulation results were obtained using the complete MB-OFDM PHY [4], including forward error correction (FEC), TFI, TDS, FDS and DCM.

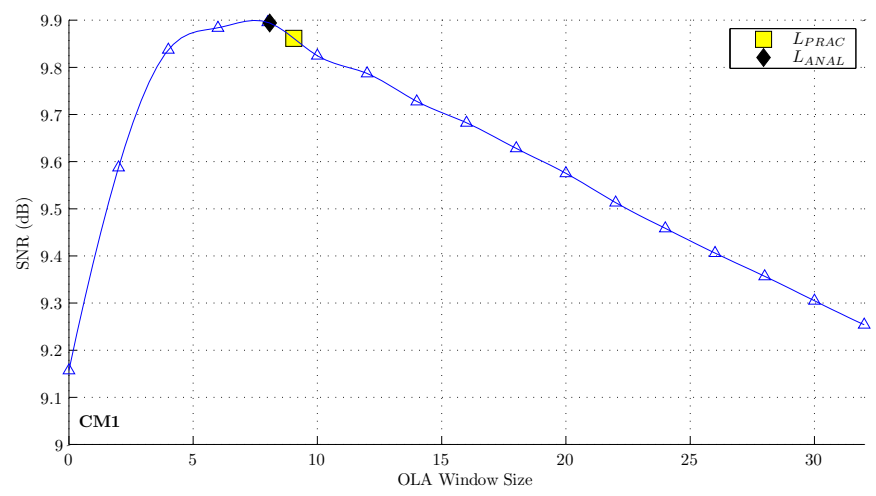

Fig. 3. SNR versus OLA size in CM1.

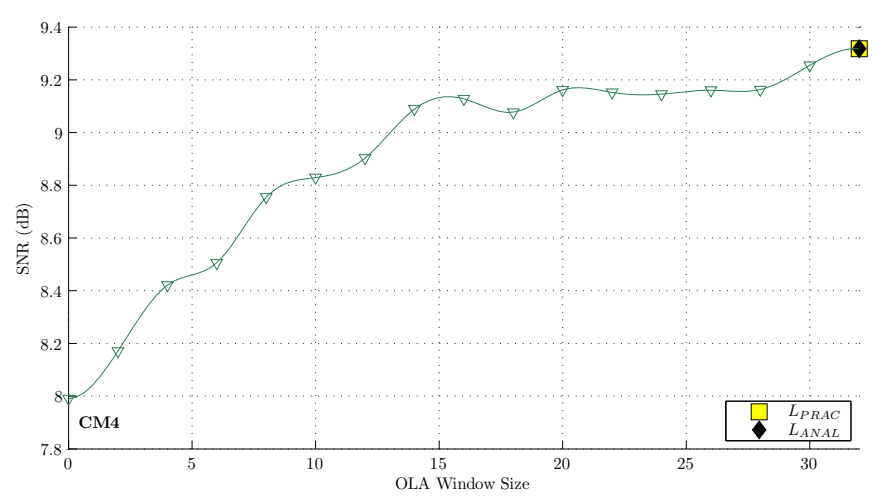

Fig. 4. SNR versus OLA size in CM4.

\section{A. Accuracy of OLA Estimation}

In Sections II and III respectively, we derived a theoretical expression (14) and practical expression (19) to estimate the optimal OLA size for a given CIR.

Figs. 3 and 4 show the effective post-OLA SNR for any arbitrary CIR from CM1 and CM4 respectively. The nominal $\mathrm{SNR}$, at the input to the receiver baseband, is $10 \mathrm{~dB}$ with any lost energy due to receiver's truncation of the CIR. In the long CM4 CIR of Fig. 4, the optimal OLA uses all 32 samples of the ZP. In this case, where both the analytic and practical OLA size estimations schemes correctly identify that the peak SNR is obtained by using the entire $\mathrm{ZP}$, there is no gain by having an adaptive OLA. However, in the shorter CM1 CIR of Fig. 3 , the optimal OLA uses only 4 samples of the ZP. In other words, an adaptive OLA would allow 28 samples of the ZP to be discarded and thereby improve the post-equalizer SNR by up to $0.7 \mathrm{~dB}$.

The radius of the circles in Fig. 5 are proportional to the probability of obtaining a given $L_{P R A C}$ for an ideal OLA size $L_{A N A L}$. The expected values are also shown. We observe that $L_{P R A C}$ tends to overestimate and underestimate $L_{A N A L}$ for small and large OLA sizes respectively. Fortunately, as shown in Figs. 3 and 4, this error is small enough that it does not significantly impact on the effective SNR. In other words, a few samples either side of the peak is of little consequence. 


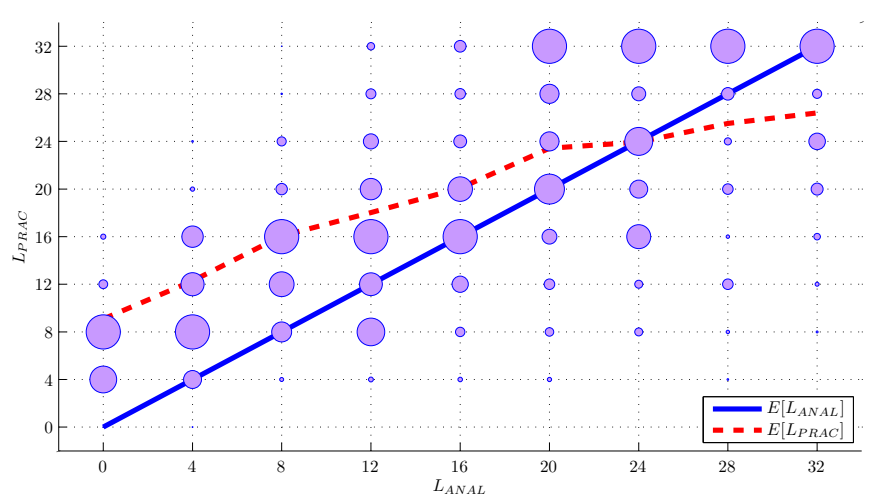

Fig. 5. Comparison of practical and analytical optimal sizes.
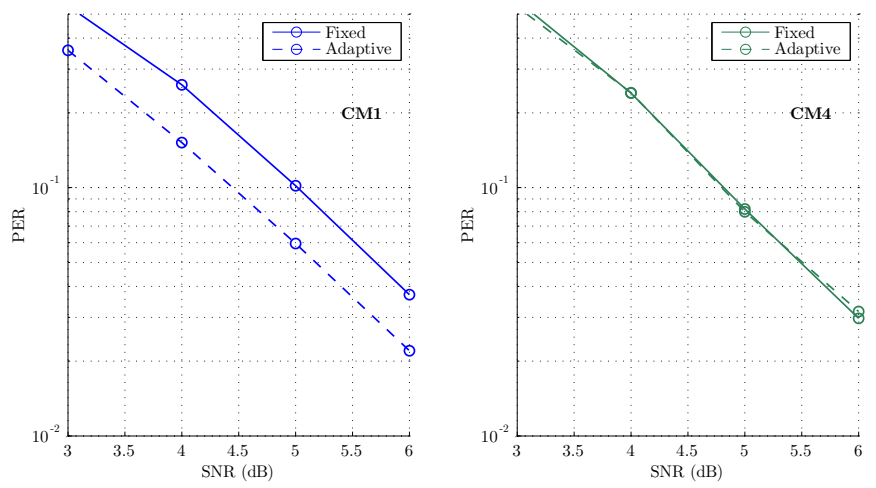

Fig. 6. PER versus SNR at input to digital baseband.

\section{B. Impact on PER}

Fig. 6 shows the impact of an adaptive OLA on PER. These simulations use 1000 byte packets transmitted at $200 \mathrm{Mb} / \mathrm{s}$ with each SNR point obtained over 5,000 unique channels. It can be seen that the adaptive OLA, based on the simplified estimate of (19), outperforms or matches a fixed OLA of 32 samples in all cases. Since longer channels have more energy in the $\mathrm{ZP}$, the higher order channel models offer less improvement since their optimal OLA size is closer to the 32 sample maximum. In other words, CM1 channels experience an average improvement of $0.6 \mathrm{~dB}$ whereas CM4 channels show no discernable difference. This confirms an intuitive expectation that an adaptive OLA is advantageous in all but extremely long channels.

As discussed previously, the maximum OLA size $L$ can be 32 samples, 37 samples or a combination of the two. If we consider the probability distribution of Fig. 2, we can expect that $50 \%$ of CM4 channels would benefit by some small degree if we could take advantage of the 5 samples in the OLA window when the TFC permits. This is confirmed in Fig. 7 where the impact of different maximum OLA sizes on the CM4 PER are shown. When the PER is $10 \%$, a gain of 0.12 $\mathrm{dB}$ can be obtained by using an OLA of 37 samples on every second symbol. This means that TFC 3 or 4 are to be preferred for long range operation since they allow a larger OLA every

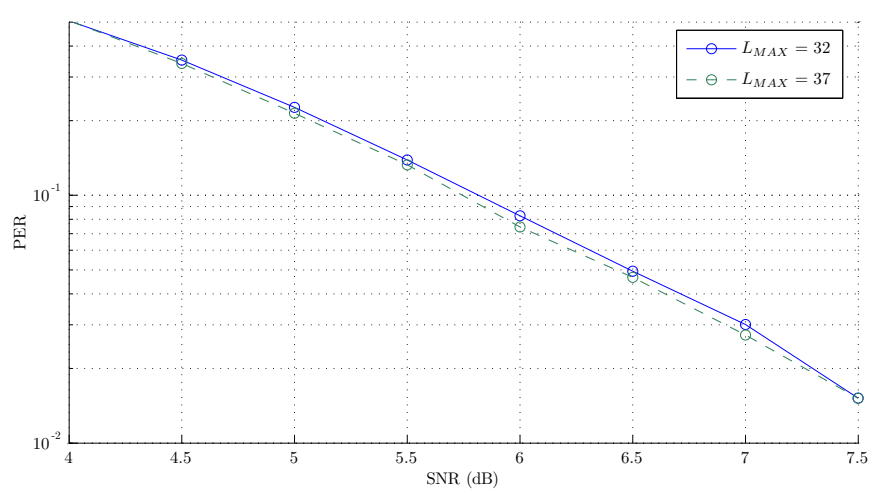

Fig. 7. PER versus SNR for different maximum ZP lengths.

second symbol. Although the performance could potentially be better again with TFC 5, 6 or 7, where the maximum OLA would always be 37 samples, the FCC-mandated reduction in power leads to more loss than gain.

\section{CONCLUSION}

In this paper, we analytically determined a bound on the effective post-equalizer SNR as a function of CIR and OLA size. We also developed a low-complexity estimator suitable for real-time implementation. Simulation results using the emerging MB-OFDM UWB standard showed that gains of up to $0.6 \mathrm{~dB}$ in multipath channels could be obtained by using an adaptive OLA. It was also shown an adaptive OLA allowed TFCs 3 and 4 to offer a slight advantage in extreme longrange operation since the lack of frequency hopping effectively increases the size of the ZP.

\section{REFERENCES}

[1] K. Siwiak and D. McKeown, Ultra-wideband Radio Technology. Chichester, England: Wiley and Sons, 2004.

[2] First Report and Order, Revision of Part 15 of the Commission's Rules Regarding Ultra-Wideband Transmission Systems, Federal Communications Commission ET Docket 98-153, Feb. 2002.

[3] A. Batra, J. Balakrishnan, G. R. Aiello, J. R. Foerster, and A. Dabak, "Design of a multiband OFDM system for realistic UWB channel environments," in IEEE Transactions on Microwave Theory and Techniques, vol. 52, no. 9, Sept. 2004, pp. 2123-2138.

[4] High Rate Ultra Wideband PHY and MAC Standard, ECMA International ECMA-368, Dec. 2005.

[5] O. Osterby, "The SVD and linear equations," University of Aarhus, Tech. Rep., Nov. 2000.

[6] Z. Zhang and L. Lai, "A novel OFDM transmission scheme with lengthadaptive cyclic prefix," Journal of Zhejiang University Science, vol. 5, no. 11, pp. 1336-1342, 2004.

[7] Z. Wang and G. B. Giannakis, "Wireless multicarrier communications," IEEE Signal Processing Magazine, vol. 17, no. 3, pp. 29-48, 2000.

[8] B. Muquet, Z. Wang, G. B. Giannakis, M. de Courville, and P. Duhamel, "Cyclic prefixing or zero padding for wireless multicarrier transmissions," IEEE Transactions on Communications, vol. 50, no. 12, pp. 2136-2148, 2002.

[9] A. F. Molisch, J. R. Foerester, and M. Pendergrass, "Channel models for ultrawideband personal area networks," IEEE Wireless Commun. Mag. pp. 14-21, Dec. 2003.

[10] A. Saleh and R. Valenzuela, "A statistical model for indoor multipath radio propogation channels," IEEE J. Select Areas Commun., vol. SAC5, pp. $128-137$, Feb. 1987 